Please do not remove this page

RMIT

UNIVERSITY

\title{
A laser obstacle warning and avoidance system for unmanned aircraft sense-and-avoid
}

Sabatini, Roberto; Gardi, Alessandro; Ramasamy, Subramanian

https://researchrepository.rmit.edu.au/esploro/outputs/9921862297101341/filesAndLinks?institution=61RMIT_INST\&index=null

Sabatini, R., Gardi, A., \& Ramasamy, S. (2014). A laser obstacle warning and avoidance system for unmanned aircraft sense-and-avoid. Applied Mechanics and Materials, 629, 355-360.

https://doi.org/10.4028/www.scientific.net/AMM.629.355

Document Version: Accepted Manuscript

Published Version: https://doi.org/10.4028/www.scientific.net/AMM.629.355

Repository homepage: https://researchrepository.rmit.edu.au

(C) 2014 Trans Tech Publications

Downloaded On 2023/04/26 23:01:30 +1000

Please do not remove this page 
Thank you for downloading this document from the RMIT Research Repository.

The RMIT Research Repository is an open access database showcasing the research outputs of RMIT University researchers.

RMIT Research Repository: http://researchbank.rmit.edu.au/

\section{Citation:}

Sabatini, R, Gardi, A and Ramasamy, S 2014, 'A laser obstacle warning and avoidance system for unmanned aircraft sense-and-avoid', Applied Mechanics and Materials, vol. 629, pp. 355-360.

See this record in the RMIT Research Repository at:

https://researchbank.rmit.edu.au/view/rmit:24985

Version: Accepted Manuscript

Copyright Statement: (C) 2014 Trans Tech Publications

Link to Published Version:

http://dx.doi.org/10.4028/www.scientific.net/AMM.629.355 


\title{
A Laser Obstacle Warning and Avoidance System for Unmanned Aircraft Sense-and-Avoid
}

\author{
Roberto Sabatini ${ }^{1, a}$, Alessandro Gardi ${ }^{1}$ and Subramanian Ramasamy ${ }^{1}$ \\ ${ }^{1}$ School of Aerospace, Mechanical and Manufacturing Engineering \\ RMIT University, Melbourne, VIC 3000, Australia \\ aroberto.sabatini@rmit.edu.au
}

Keywords: LIDAR, Low-Level Flight, Obstacle Detection, Obstacle Warning, Obstacle Avoidance, Sense-and-Avoid, Uncertainty volume and Unmanned Aircraft.

\begin{abstract}
This paper presents an overview of the research activities performed to develop a new scaled variant of the Laser Obstacle Avoidance and Monitoring (LOAM) system for small-tomedium size Unmanned Aircraft (UA) platforms. This LOAM variant $\left(\mathrm{LOAM}^{+}\right)$is proposed as one of the non-cooperative sensors employed in the UA Sense-and-Avoid (SAA) system. After a brief description of the LOAM system architecture, the mathematical models developed for obstacle avoidance and calculation of alternative flight path are presented. Additionally, a new formulation is adopted for defining the uncertainty volumes associated with the detected obstacles. Simulation case studies are carried out to evaluate the performances of the avoidance trajectory generation and optimisation algorithms, which demonstrate the ability of $\mathrm{LOAM}^{+}$to effectively detect and avoid fixed low-level obstacles in the intended path.
\end{abstract}

\section{Introduction}

In recent years, laser-based Light Detection and Ranging (LIDAR) has become a promising technology for navigation in Unmanned Aircraft (UA). The adoption of LIDAR for a number of civil and military applications [1] is attributed to its good detection performance, outstanding angular resolution and range accuracy. The widespread adoption of small-to-medium size UA designed for low-level or nap-of-the-earth flight applications has resulted in raising public concerns regarding the overall safety of people and property on the ground. Low visibility due to bad weather or natural/man-made obscurants is the primary factor responsible for a number of collisions with fixed obstacles in low-level operations of UA. In this context, obstacle detection, warning and avoidance is of paramount importance and suitable algorithms are necessary for carrying out UA mission- and safety-critical tasks. The Laser Obstacle Avoidance and Monitoring (LOAM) system was previously developed and tested on rotary-wing platforms [2-5], and the development of an UA version is currently being performed [6,7]. To gain unrestricted access to airspace, current research activities are focussing on developing an effective and certifiable Sense-and-Avoid (SAA) system incorporating both non-cooperative sensors and cooperative systems. Significant research efforts are currently being carried out to develop cooperative, non-cooperative and hybrid architectures capable of achieving this fundamental goal [8]. The proposed LOAM variant for $\mathrm{UA}\left(\mathrm{LOAM}^{+}\right)$is adopted as a non-cooperative SAA sensor. $\mathrm{LOAM}^{+}$detects potentially dangerous obstacles placed in or nearby the nominal UA flight trajectory, classifies the detected obstacles, and provides suitable steering data to the UA flight control systems, as well as timely caution/warnings to the ground crew (both aural and visual), in order to implement effective avoiding manoeuvres.

\section{UA Laser Obstacle Avoidance and Monitoring System}

The LOAM system requirements include reliable detection of all ground obstacles within a suitable volume around the aircraft (adjustable as a function of platform dynamics), in all-weather conditions and in day/night operations. The LOAM laser beam scans periodically the area around the nominal flight trajectory inside a Field of View (FOV) of $40^{\circ}$ in azimuth and $30^{\circ}$ in elevation, 
with an adjustable Field of Regard (FOR) capability of $\pm 20^{\circ}$ both on azimuth and elevation, centred on the optical axis of the system. During every scan the laser beam is scanned with an elliptical pattern [7] in the FOV of the system. The most dangerous obstacles including wires, poles, buildings, etc. are detected by the system. Dedicated signal processing algorithms are implemented as part of the LOAM to detect the low-level obstacles independent from the platform motion. The shapes of the obstacles are reconstructed without using any additional navigation data in slowmoving platforms. In platforms with high dynamics envelopes, the measurements from LOAM are integrated with other UA navigation sensors as part of an integrated navigation and guidance system [9]. LOAM performs echo detection through analogue signal processing that comprises of an optical-electrical conversion, a signal pre-amplification and a threshold comparison. Reliable obstacle detection is performed by local analysis on single echoes and thus the range, angular coordinates and other features of the obstacle are obtained. Successively, global analysis is performed in order to group the echoes detected during a scan period and to reconstruct the shape of the obstacles. A typical LOAM integrated architecture is illustrated in Fig. 1. LOAM main components are the Sensor Head Unit (SHU), the Control Panel (CP) and the Display Unit (DU). The SHU generates laser beam scans, detects return echoes, analyses these echoes in order to compute range, coordinates and local geometrical characteristics of the obstacles and then communicates to the LOAM Processing Unit (PU). LOAM employs three key algorithms namely; calculation of the future trajectory, calculation of the intersections with the detected obstacles and determination of the optimal flight path from a set of avoidance trajectories in case a risk of collision is determined. The PU interacts with the UA Flight Management System (FMS) [10, 11], which encompasses the integrated navigation and guidance system [9] and the SAA system [8]. Unmanned Aerial System (UAS) datalink serves as the communication medium between UA and ground systems. The CP and DU are adopted based on the specific unmanned platform employed.

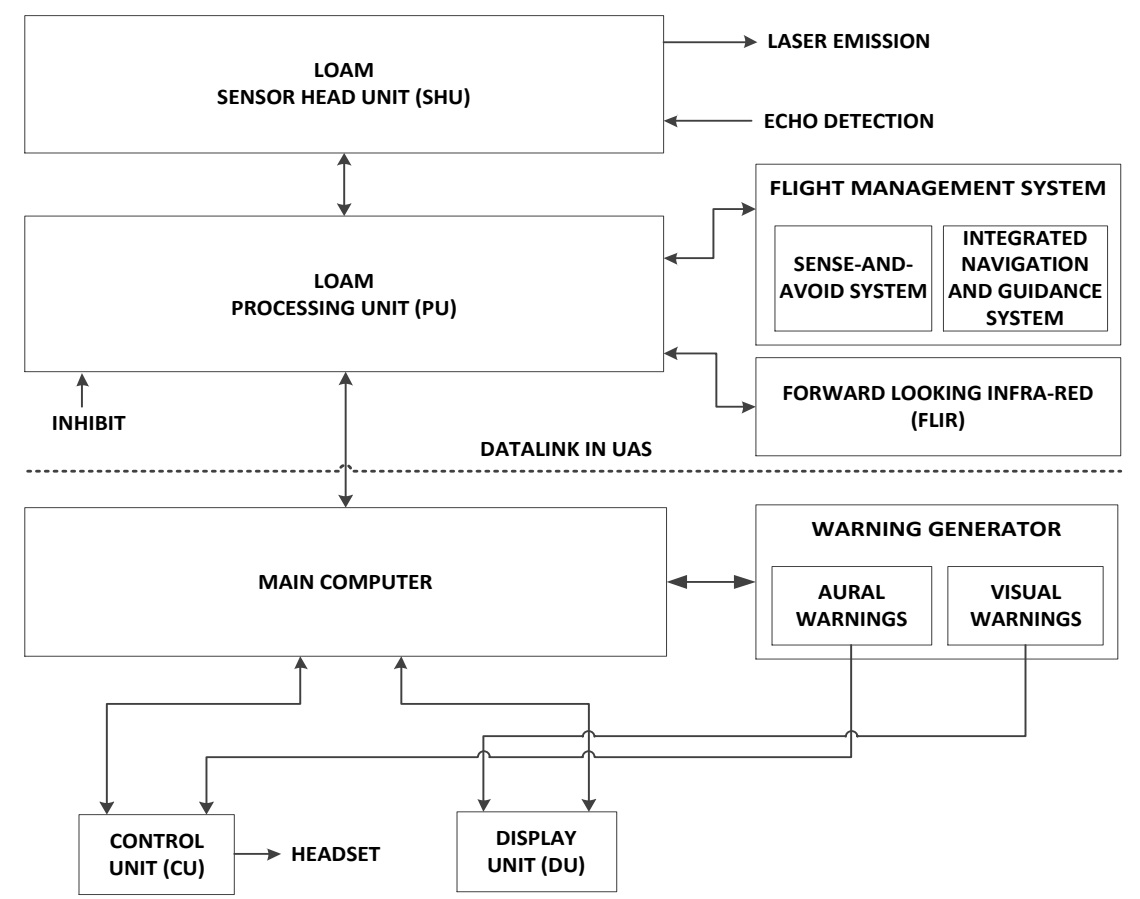

Fig. 1. Typical LOAM integration architecture.

\section{Mathematical Models}

A number of obstacles including wires, tress and extended structures are detected by LOAM. The algorithms for LOAM obstacle detection and performance estimation are described in [5-7]. After the obstacles are detected and the risk of collision is determined, avoidance trajectory 
generation algorithm is triggered to determine the necessary manoeuvres for safe avoidance of obstacles in case collisions are predicted. The approximated three-degree-of-freedom (3-DoF) dynamics model of the fixed-wing UA platform adopted in the $\mathrm{LOAM}^{+}$avoidance trajectory generation algorithm is based on the assumptions of a point-mass rigid body, inertial reference system centred on the initial position of the UA point-mass, constant gravitational acceleration $\left(g=9.81 \mathrm{~ms}^{-2}\right)$ and constant mass along the avoidance trajectory. Furthermore, the UA True Air Speed (TAS), $v$, is expressed as a tangent to the aircraft trajectory and the wind components are included along the $\{x, y, z\}$ axes as a vector given by $v_{w}=\left\{v_{W_{x}}, v_{W_{y}}, v_{W_{z}}\right\}$. The system of differential equations for the UA dynamics is given by:

$$
\begin{aligned}
\dot{x} & =v \cdot \cos \gamma \cdot \sin \chi+v_{w_{x}} \\
\dot{y} & =v \cdot \cos \gamma \cdot \cos \chi+v_{w_{y}} \\
\dot{z} & =v \cdot \sin \gamma+v_{w_{z}} \\
\dot{v} & =\frac{T-D}{m}-g \cdot \sin \gamma \\
\dot{\gamma} & =\frac{g}{v} \cdot(N \cdot \cos \mu-\cos \gamma) \\
\dot{\chi} & =\frac{g}{v} \cdot \frac{N \cdot \sin \mu}{\cos \gamma}
\end{aligned}
$$

where $T$ is the propulsive thrust $[\mathrm{N}] ; D$ is the aerodynamic drag force $[\mathrm{N}] ; \gamma$ is the flight path angle $\left[{ }^{\circ}\right] ; \chi$ is the track angle $\left[{ }^{\circ}\right]$ and $\mu$ is the bank angle $\left[{ }^{\circ}\right]$. During the avoidance manoeuvre, the load factor $N$ is set close but below to the certified flight envelope limits of the UA (i.e., $N=2$ ). The assumed initial TAS in this case is $v=25 \mathrm{~ms}^{-1}$. Assuming that during the avoidance manoeuvre the UA control system provides a linear variation of $\mu$ up to the assumed maximum bank angle, $\mu_{M A X}$, $\mu$ and its derivative are given by:

$$
\begin{cases}\mu=\mu_{0}+\dot{\mu}_{M A X} \cdot t & \left(\mu \leq \mu_{M A X}\right) \\ \dot{\mu}=0 & \left(\mu=\mu_{M A X}\right)\end{cases}
$$

where $\mu_{0}$ is the bank angle with respect to $t=0$. The maximum bank angle is expressed as:

$$
\mu_{M A X}=\operatorname{acos}\left(\frac{1}{N_{M A X}}\right)
$$

In this case, the maximum roll rate is $\dot{\mu}_{M A X}=20 \%$. When the distance between two detected obstacles is comparable with the calculated uncertainty values, or with the UA dimensions, the two obstacles are combined in a single avoidance volume. After a set of safe trajectories is generated, the optimal trajectory is selected and fed to the aircraft guidance subsystem. The implemented decision logic is based on minimisation of the cost function:

$$
J=w_{t} \cdot t_{S A F E}+w_{f} \int[S F C \cdot T(t)] d t-w_{d} \cdot D_{\min }-w_{i d} \cdot \int D(t) d t
$$

where $J$ is the cost function, $D(t)$ is the estimated distance of the generated avoidance trajectory points from the avoidance volume associated with the obstacle, $D_{\min }=\min [D(t)]$ is the estimated minimum distance of the avoidance trajectory from the avoidance volume, $t_{S A F E}=\left.t\right|_{D_{\min }}$ is the time at which the safe avoidance condition is successfully attained, $S F C[\mathrm{~kg} / \mathrm{N} / \mathrm{s}]$ is the specific fuel consumption, $T(t)$ is the thrust profile and $\left\{w_{t}, w_{f}, w_{d}, w_{i d}\right\}$ are the weightings attributed to time, fuel, distance and integral distance respectively. The active obstacle set always includes the obstacles in the current FOV and also the ones previously detected and recorded by the LOAM history function, described in [5-7]. Error analysis is performed to determine the overall uncertainty volume surrounding the detected obstacles. The uncertainties in the UA position, velocity and attitude are due the errors in the navigation state vector parameters and the uncertainties in the tracking measurements are due to the $\mathrm{LOAM}^{+}$sensor errors. Both the UA navigation uncertainty and the obstacle tracking uncertainty volumes are described using ellipsoidal bounding surfaces [8]: 


$$
\frac{x^{2}}{\sigma_{x}^{2}}+\frac{y^{2}}{\sigma_{y}^{2}}+\frac{z^{2}}{\sigma_{z}^{2}}=1
$$

The uncertainty associated with the current position of the UA, $\left(\sigma_{x}, \sigma_{y}, \sigma_{z}\right)$ depends on the standard deviations of the UA dynamics parameters given by $\left\{\sigma_{v}, \sigma_{\gamma}, \sigma_{\mu}, \sigma_{y}, \sigma_{v_{W_{x}}}, \sigma_{v_{W_{y}}}, \sigma_{v_{W_{z}}}\right\}$ [11]. The state vector of the UA is expressed as:

$$
x(t)=x\left(x_{i}\left(t_{0}\right), u_{i}\left(t_{0}\right), p_{k}, t\right)=x\left(p_{j}\right)
$$

where $x_{i}\left(t_{0}\right)$ and $u_{i}\left(t_{0}\right)$ are the state vector and controls defined at time, $t_{0}, t$ is the time epoch, $i$ is the number of variables in the state vector, $p_{k}$ is the augmentative vector, $k$ is the sum of $j$ solved parameters of $x_{i}\left(t_{0}\right)$ and $l$ number of additional parameters. The coefficients of the spherical harmonic decomposition are used to describe the navigation and tracking uncertainties and they are introduced as additional parameters in the state vector described in Eq. 11. The coefficients of the spherical harmonic function $L_{n, m}$ are given by:

$$
\begin{aligned}
& L_{n, m}=\left\{C_{n, o}, C_{n, m} \text { and } S_{n, m}\right\} \\
& S_{n, m}=0 ; n, m \varepsilon \mathrm{N} \\
& C_{n, m}=0 ; n, m \varepsilon(2 \mathrm{~N}+1)
\end{aligned}
$$

and for all other $n, m$ :

$$
C_{n, m}=\frac{3}{a^{l}} \frac{\left(\frac{n}{2}\right) !\left(\frac{n}{m}\right) !\left(2-\delta_{n m}\right)}{2^{m}(n+3)(n+1) !} \times \sum_{i=0}^{(n-m) / 4} \frac{\left(a^{2}-b^{2}\right)^{(m+4 i) / 2}\left[c^{2}-\left(\frac{1}{2}\right)\left(a^{2}-b^{2}\right)\right]^{(l-m-4 i) / 2}}{16^{i}\left(\frac{n-m-4 i}{2}\right) !\left(m+\frac{2 i}{2}\right) ! i !}
$$

where $\delta_{n m}$ is the Kronecker symbol and $(a, b, c)$ represents the semi-major radius of the avoidance volume ellipsoid. For error estimations, the variation in the state vector is expressed as:

$$
\delta\left(x_{i}(t)\right)=\left[\frac{\delta x_{i}}{\delta p_{j}}\right]_{t} \cdot \sigma_{p_{j}}
$$

The measurements from $\mathrm{LOAM}^{+}$in terms of range $(R)$, elevation $(E L)$, and azimuth $(A Z)$ measurements of the obstacles are expressed as:

$$
\begin{aligned}
& x\left(t_{j}\right)=R\left(t_{j}\right) \cos \left(E L\left(t_{j}\right)\right) \cos \left(A Z\left(t_{j}\right)\right) \\
& y\left(t_{j}\right)=R\left(t_{j}\right) \cos \left(E L\left(t_{j}\right)\right) \sin \left(A Z\left(t_{j}\right)\right) \\
& z\left(t_{j}\right)=-R\left(t_{j}\right) \sin \left(E L\left(t_{j}\right)\right)
\end{aligned}
$$

The measurements in spherical coordinates $(M)$ are converted to an inertial system given by:

$$
M=M\left(R, A Z, E L\left(E L_{i}, \omega_{E}, t\right)\right)
$$

where $E L_{i}$ is the inertial longitude and $\omega_{E}$ is the rotation rate of the Earth. After obtaining the navigation and the avoidance volume ellipsoids, they are translating to unified range and bearing uncertainty descriptors and expressed as spherical harmonic coefficients as in Eq. 12. The range and bearing tracking errors associated with the obstacles are determined as $\sigma_{\text {range }(x, y, z)}, \sigma_{\text {azimuth }(x, y, z)}$ and $\sigma_{\text {elevation }(x, y, z)}$. Finally, the total uncertainty volume is obtained by inflating the tracking error volume with the UA navigation error components [8]. The Time-to-Collision $\left(t_{T T C}\right)$ is expressed with respect to the collision probability as:

$$
\operatorname{Pr}(C)=\operatorname{Pr}\left(\left|D_{\min }\left(t_{T T C}\right)\right|<V\right)
$$

where $\operatorname{Pr}(C)$ is the probability of collision with the uncertainty volume, $V$. In order to assure adequate safety levels, a separation buffer is introduced, which inflates the avoidance volume associated with the obstacle. In particular, to provide a confidence level of $95 \%$, the uncertainty volume is calculated using twice the standard deviations (i.e. two-sigma) of both obstacle tracking and UA navigation errors. 


\section{Simulation and Results}

Simulation activities were performed in realistic test scenarios to assess the performance and validate the avoidance trajectory generation and optimisation algorithm described for small size UA. An example of these test cases, including a nose mounted $\mathrm{LOAM}^{+}$on the AEROSONDE UA and various man-made obstacles, is illustrated in Fig. 2 (a). The AEROSONDE is approaching a power transmission line consisting of an electricity pylon and a number of wires on its side. Additionally, it is assumed that the height of the pylon is exceeded by a building obstacle behind it and within the LOAM ${ }^{+}$FOV. The lateral wires on the left and right side of the pylon are combined in two separate avoidance volumes. The pylon and the building form the third and fourth avoidance volumes. The four ellipsoidal tracking error volumes and the UA navigation error ellipsoid are determined and combined. The overall uncertainty volume is obtained by inflating the four avoidance volumes separately with the navigation error components of the UA assuming, in this particular test case, uncorrelated error statistics. Since the algorithm ascertain that the original UA flight trajectory leads to a collision, a set of feasible and non-conflicting avoidance trajectories is generated in real-time (these trajectories are obtained by attributing various weightings to time, fuel, distance and integral distance respectively). The total resulting uncertainty volume and the generated avoidance trajectories are illustrated in Fig. 2 (b).

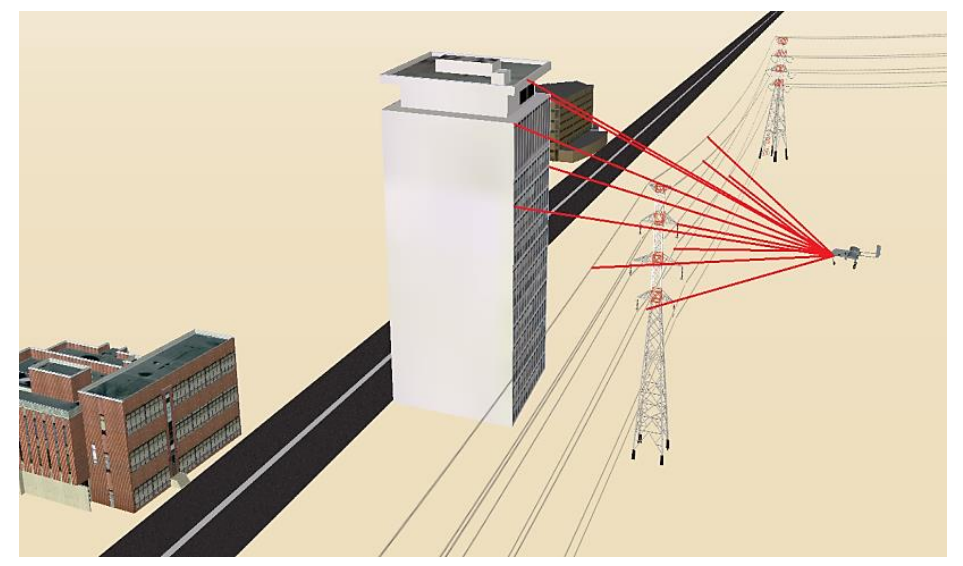

(a) Case study scenario

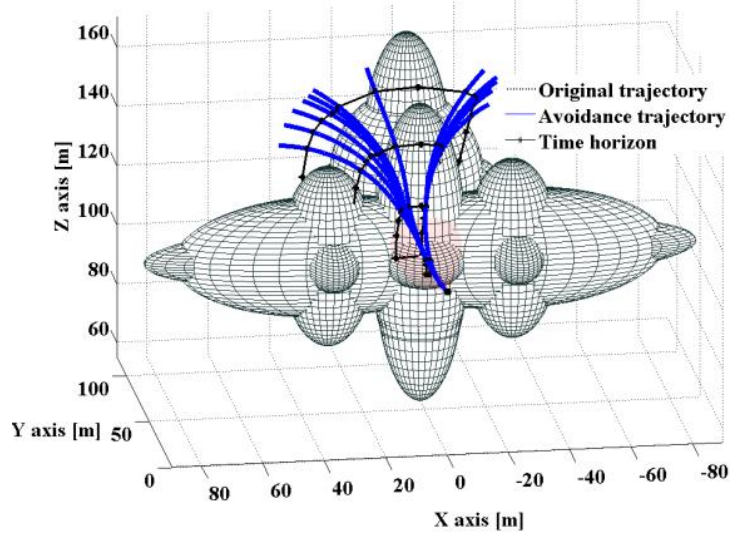

(b) Avoidance trajectories

Fig. 2. Simulation case.

\section{Conclusions and Future Work}

A scaled variant of the Laser Obstacle Avoidance and Monitoring (LOAM) system for use in small-to-medium size Unmanned Aircraft (UA) was presented $\left(\mathrm{LOAM}^{+}\right)$. The research focused on the possible adoption of $\mathrm{LOAM}^{+}$as part of an integrated avionics architecture for non-cooperative Sense-and-Avoid (SAA). The algorithms for avoidance trajectory generation/optimisation and overall uncertainty volume determination were described and some simulation results were presented. Current research activities are addressing the integration of $\mathrm{LOAM} \mathrm{LOAM}^{+}$with Forward Looking Sensors (FLS) and Night Vision Imaging Systems (NVIS) [12]. Display formats currently being developed for the UA remote pilot station include a Safety Line (SL) format, a Wires \& Poles (WP) format, an All Obstacles (AO) format and an Integrated LOAM/FLS (ILF) format. Additional mathematical descriptors including covariant and contravariant tensors are being adopted for uncertainty volume determination, toward a unified analytical approach covering both cooperative and non-cooperative SAA applications. The possible integration of LIDAR with other UA avionic sensors is being studied and future research will address the SAA functionalities required for 4-Dimensional Trajectory Based Operations (4D-TBO) [10, 11, 13]. 


\section{References}

[1] Unmanned Aircraft System (UAS) Service Demand 2015-2035, Technical Report, United States Air Force, US Department of Transportation. (2013)

[2] R. Sabatini, Tactical Laser Systems Performance Analysis in Various Weather Conditions, in RTO-MP-001 - E-O Propagation, Signature and System Performance under Adverse Meteorological Conditions Considering Out of Area Operations, Sensors and Electronics Technology (SET) panel, NATO Research and Technology Organization (RTO), Naples, Italy, 1998, pp. 29-1 to 29-13.

[3] R. Sabatini, F. Guercio, and S. Vignola, Airborne Laser Systems Performance Analysis and Mission Planning, in RTO-MP-046 - Advanced Mission Management and Systems Integration Technologies for Improved Tactical Operations, Systems Concepts and Integration (SCI) panel, NATO Research and Technology Organization (RTO), Florence, Italy, 1999.

[4] R. Sabatini, E. Roviaro and M. Cottalasso, Development of a Laser Collision Avoidance System for Helicopters: Obstacle Detection/Classification and Calculation of Alternative Flight Paths, in RTO-MP-092 - Complementarities of LADAR and RADAR, Sensors \& Electronics Technology Panel (SET), NATO Research and Technology Organization (RTO), Prague, Czech Republic, 2002.

[5] R. Sabatini and M. A. Richardson, RTO AGARDograph AG-300 Vol. 26: Airborne Laser Systems Testing and Analysis, NATO Science and Technology Organization, 2010.

[6] R. Sabatini, A. Gardi and M.A. Richardson, LIDAR Obstacle Warning and Avoidance System for Unmanned Aircraft, International Journal of Mechanical, Industrial Science and Engineering, vol. 8, pp. 62-73. (2014)

[7] R. Sabatini, A. Gardi and S. Ramasamy, A Laser Obstacle Warning and Avoidance System for Manned and Unmanned Aircraft, in proceedings of IEEE International Workshop on Metrology for Aerospace, Benevento, Italy, pp. 616-621. (2014)

[8] S. Ramasamy, R. Sabatini and A. Gardi, Avionics Sensor Fusion for Small Size Unmanned Aircraft Sense-and-Avoid, in proceedings of IEEE International Workshop on Metrology for Aerospace, Benevento, Italy, pp. 271-276. (2014)

[9] R. Sabatini, S. Ramasamy, A. Gardi, and L. Rodriguez Salazar, Low-Cost Sensors Data Fusion for Small Size Unmanned Aerial Vehicles Navigation and Guidance, International Journal of Unmanned Systems Engineering, 1(3), pp. 16-47. (2013) DOI: 10.14323/ijuseng.2013.11

[10] S. Ramasamy, R. Sabatini, A. Gardi and Y. Liu, Novel Flight Management System for RealTime 4-Dimensional Trajectory Based Operations, in proceedings of AIAA Guidance, Navigation \& Control Conference, Boston, USA. (2013) DOI: 10.2514/6.2013-4763

[11]S. Ramasamy, R. Sabatini, A. Gardi and T. Kistan, Next Generation Flight Management System for Real-Time Trajectory Based Operations, Applied Mechanics and Materials (Special Issue: AEROTECH V, Kuala Lumpur, Malaysia). (2014)

[12]R. Sabatini, M. A. Richardson, M. Cantiello, M. Toscano and P. Fiorini, A Novel Approach to Night Vision Imaging Systems Development, Integration and Verification in Military Aircraft, Aerospace Science and Technology, 31(1), pp. 10-23. (2013) DOI: 10.1016/j.ast.2013.08.021

[13]A. Gardi, R. Sabatini, S. Ramasamy and K. de Ridder, 4-Dimensional Trajectory Negotiation and Validation System for the Next Generation Air Traffic Management, in proceedings of AIAA Guidance, Navigation \& Control Conference, Boston, USA. (2013) DOI: 10.2514/6.2013-4893 